\section{CircRNAs in lifespan}

Circular RNAs (circRNAs) are for the most part generated by backsplicing of exons in protein-coding genes. They have been discovered in many species, but only few have been functionally characterized. Weigelt et al. now find that the circRNA generated by the sulfateless gene (circSfl) controls the lifespan of flies.

The nutrient-sensing insulin/insulin-like growth factor signalling pathway is a key regulator of metabolism and ageing. As inhibition of this pathway has been shown to extend lifespan,

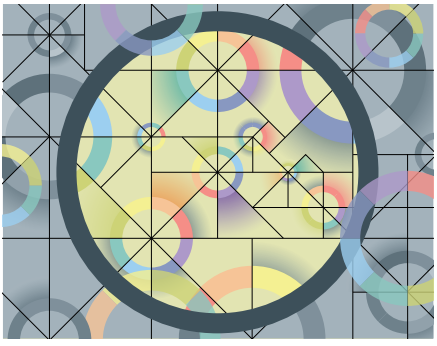

the authors went on to analyse the links between insulin-mediated lifespan extension and circRNAs.

Whereas global circRNA expression increased with age in brain tissue of wild-type Drosophila melanogaster, the increase was significantly lower in the brain of long-lived dilp 2-3,5 mutant flies (which are defective in the expression of insulinlike peptides). This observation suggests that ageing-dependent accumulation of circRNAs is slowed down by reduced insulin signalling. The authors focused on characterizing circSfl, as it was strongly upregulated in dilp 2-3,5 mutant flies and other long-lived insulin mutants and is regulated independently of its cognate gene.

When experimentally overexpressed in wild-type flies, the ubiquitous expression of circSfl led to up to $15 \%$ increase in median lifespan. The generation of circSfl was linked with the expression of one of two alternative splicing isoforms of the cognate, linear mRNA ( $S f l R A$ ), and when expression of circSfl and $S f l R A$ were reduced in insulin-mutant flies, the effect on lifespan was abolished. Thus, the insulin-mediated lifespan extension requires circSfl and Sfl RA.

circSfl was found to be translated in vivo, and circSfl protein levels increased significantly in dilp 2-3,5 mutants. Moreover, overexpression of the circSfl ORF from a linear transcript extended lifespan of female flies, together indicating that the protein encoded by circSfl is sufficient to increase lifespan. The sfl linear transcripts encode a Golgi enzyme that catalyses the synthesis of heparan sulfate, whereas the circSfl protein is a truncated version that lacks catalytic activity. Interestingly, the full length protein induced lifespan extension only if it was overexpressed specifically in neurons, suggesting that the two proteins might have both common and unique roles in ageing.

This study shows that increased expression of circSfl and linear $\mathrm{sl}$, and of the proteins they encode, can extend lifespan in flies. It will be interesting to elucidate the mechanisms leading to longer lifespan and how the different transcripts and proteins interact.

Kim Baumann

ORIGINAL ARTICLE Weigelt, C. M. et al. An insulin-sensitive circular RNA that regulates lifespan in Drosophila. Mol. Cell https://doi.org/10.1016/j.molcel.2020.06.011 (2020) RELATED ARTICLE Chen, L. The expanding regulatory mechanisms and cellular functions of circular RNAs. Nat. Rev. Mol. Cell Biol. https://doi.org/10.1038/s41580-0200243-y (2020)

\section{Journal Club}

\section{LIPID RAFTS COME OF AGE}

Biological membranes are partitioned across a variety of length scales from multi-component molecular complexes to micron-scale domains in polarized cells. Between these regimes is the mesoscale organization of cellular membranes into lipid-driven domains termed lipid rafts. This ultrastructural organization has fascinated and baffled a generation of membrane biologists, with persistent controversy about the nature and functions of rafts in vivo.

The raft concept proposes that cell membranes have the capacity for spontaneous self-organization into structurally and functionally distinct fluid domains, driven by subtle preferences between certain lipid classes. This concept was built on two experimental pillars. The first is the fact that biochemical fractionation of cells or tissues after lysis with non-ionic detergents yields 'detergent-resistant membrane' residues that consistently enrich for specific protein and lipid classes, implying intrinsic heterogeneity of cellular membranes. The second stems from observations that synthetic membranes composed of biomimetic lipid mixtures form beautiful, macroscopic fluid domains, owing to phase separation between ordered and disordered lipids.

Individually, these lines of evidence fell short of being truly convincing: detergent lysis creates the potential for artefactual assemblies never present in intact cells, while synthetic membranes lack the complexity and protein content of living cells. These shortcomings created confusion and doubt about the validity and value of the raft hypothesis that persists to this day. What good is all this intricate biochemistry and biophysics if it can never properly describe living membranes?

The turning point that reinvigorated the field was a remarkable paper by Baumgart et al. in 2007 showing that mammalian plasma membranes isolated as giant plasma membrane vesicles (GPMVs) — can spontaneously separate into co-existing liquid phases that organize biomembranes in striking concordance with their detergent resistance. This breakthrough was enabled by advances in confocal microscopy (Baumgart et al., 2003) applied to plasma membrane blebs isolated intact from cultured mammalian cells.

Personally, seeing these images was a moment of epiphany: I had studied similar organization in synthetic membranes for years, wondering whether it had anything to do with cells, and here it was in living colour! The synthesis of these observations with the biochemical and biophysical pillars underlying the raft concept seems obvious in hindsight. Subsequent work with GPMVs showed that the 'raft phase' is more tightly packed, more viscous, rich in saturated lipids, sterol analogues and lipidated proteins (Sezgin et al., 2016), validating the principle that ordered, lipid-driven phases can organize biomembranes. Equally important, this paper demonstrated a straightforward methodology to investigate the properties and compositions of raft domains in intact cell membranes, allowing quantitative correlations between proteins' raft affinity and their behaviour (Komura et al., 2016) and functions (Diaz-Rohrer et al., 2017) in living cells.

Ilya Levental

Department of Integrative Biology and Pharmacology, University of Texas Health Science Center at Houston, Houston, TX, USA. e-mail: ilya.levental@uth.tmc.edu

The author declares no competing interests.

ORIGINAL ARTICLES Baumgart, T. et al. Large-scale fluid/fluid phase separation of proteins and lipids in giant plasma membrane vesicles. Proc. Natl Acad. Sci. USA 104, 3165-3170 (2007)|Baumgart, T., Hess, S. T. \& Webb, W. W. Imaging coexisting fluid domains in biomembrane models coupling curvature and line tension. Nature 425, 821-824 (2003) | Diaz-Rohrer, B. B. et al. Membrane raft association is a determinant of plasma membrane localization. Proc. Natl Acad. Sci. USA 111, 8500-8505 (2014) | Komura, N. et al. Raft-based interactions of gangliosides with a GPI-anchored receptor. Nat. Chem. Biol. 12, 402-410 (2016) | Sezgin, E. et al. Elucidating membrane structure and protein behavior using giant plasma membrane vesicles. Nat. Protoc. 7 , 1042-1051 (2012)

RELATED ARTICLE Sezgin, E. et al. The mystery of membrane organization: composition, regulation and roles of lipid rafts. Nat. Rev. Mol. Cell Biol. 18, 361-374 (2017) 